\title{
Article \\ Improving Visual-Patient-Avatar Design Prior to Its Clinical Release: A Mixed Qualitative and Quantitative Study
}

\author{
Doreen J. Wetli, Lisa Bergauer, Christoph B. Nöthiger, Tadzio R. Roche $\mathbb{D}$, Donat R. Spahn, David W. Tscholl * $\mathbb{D}$ \\ and Sadiq Said
}

check for updates

Citation: Wetli, D.J.; Bergauer, L.; Nöthiger, C.B.; Roche, T.R.; Spahn, D.R.; Tscholl, D.W.; Said, S. Improving Visual-Patient-Avatar Design Prior to Its Clinical Release: A Mixed Qualitative and Quantitative Study. Diagnostics 2022, 12, 555 . https://doi.org/10.3390/ diagnostics12020555

Academic Editor: Martin Mueller

Received: 28 December 2021

Accepted: 18 February 2022

Published: 21 February 2022

Publisher's Note: MDPI stays neutral with regard to jurisdictional claims in published maps and institutional affiliations.

Copyright: (C) 2022 by the authors. Licensee MDPI, Basel, Switzerland. This article is an open access article distributed under the terms and conditions of the Creative Commons Attribution (CC BY) license (https:// creativecommons.org/licenses/by/ $4.0 /)$.
Institute of Anaesthesiology, University Hospital Zurich, Raemistrasse 100, 8091 Zurich, Switzerland; doreenjessica.wetli@usb.ch (D.J.W.); lisa.bergauer@usz.ch (L.B.); christoph.noethiger@usz.ch (C.B.N.); tadzioraoul.roche@usz.ch (T.R.R.); donat.spahn@usz.ch (D.R.S.); sadiq.said@usz.ch (S.S.)

* Correspondence: david.tscholl@usz.ch

\begin{abstract}
Visual-Patient-avatar, an avatar-based visualisation of patient monitoring, is a newly developed technology aiming to promote situation awareness through user-centred design. Before the technology's introduction into clinical practice, the initial design used to validate the concept had to undergo thorough examination and adjustments where necessary. This mixed qualitative and quantitative study, consisting of three different study parts, aimed to create a design with high user acceptance regarding perceived professionalism and potential for identification while maintaining its original functionality. The first qualitative part was based on structured interviews and explored anaesthesia personnel's first impressions regarding the original design. Recurrent topics were identified using inductive coding, participants' interpretations of the vital sign visualisations analysed and design modifications derived. The second study part consisted of a redesign process, in which the visualisations were adapted according to the results of the first part. In a third, quantitative study part, participants rated Likert scales about Visual-Patient-avatar's appearance and interpreted displayed vital signs in a computer-based survey. The first, qualitative study part included 51 structured interviews. Twenty-eight of 51 (55\%) participants mentioned the appearance of Visual-Patientavatar. In 23 of 51 (45\%) interviews, 26 statements about the general impression were identified with a balanced count of positive (14 of 26) and negative (12 of 26) comments. The analysis of vital sign visualisations showed deficits in several vital sign visualisations, especially central venous pressure. These findings were incorporated into part two, the redesign of Visual-Patient-avatar. In the subsequent quantitative analysis of study for part three, 20 of $30(67 \%)$ new participants agreed that the avatar looks professional enough for medical use. Finally, the participants identified 73\% (435 of 600 cases) of all vital sign visualisations intuitively correctly without prior instruction. This study succeeded in improving the original design with good user acceptance and a reasonable degree of intuitiveness of the new, revised design. Furthermore, the study identified aspects relevant for the release of Visual-Patient-avatar, such as the requirement for providing at least some training, despite the design's intuitiveness. The results of this study will guide further research and improvement of the technology. The study provides a link between Visual-Patient-avatar as a scientific concept and as an actual product from a cognitive engineering point of view, and may serve as an example of methods to study the designs of technologies in similar contexts.
\end{abstract}

Keywords: avatar technology; intuitiveness; patient monitoring; situation awareness; user-centred design; Visual-Patient-avatar

\section{Introduction}

The World Health Organisation (WHO) regards perioperative complications as a significant global cause of preventable death in healthcare [1]. Consequently, the guidelines for safe surgery describe continuous patient monitoring by anaesthesia providers as "extremely important" for patient safety during surgical procedures. However, healthcare 
providers must continuously perceive and interpret many monitoring values to gain and maintain situation awareness, and initiate possibly lifesaving therapies. The central concept of well-informed decisions is situation awareness, defined as perceiving and understanding the current situation, including its expected course [2-4]. Failure to perceive principally available information is more common than misinterpreting information or inferring incorrect projections from it [5]. Research showed that up to eighty percent of adverse events in anaesthesia result from impaired situation awareness [6].

Although these problems are well known and the design of patient monitoring holds opportunities to increase situational awareness [7], there have been no significant improvements in information presentation since the mid-20th century [8]. Current patient monitoring relies on numbers and waveforms, which humans can only read one-by-one, and can have difficulty memorising and integrating mentally $[3,7,9]$. The resulting delay in understanding clinical situations [7] calls for a new monitoring approach.

\section{Visual-Patient-Avatar}

The Visual-Patient-avatar is a visualisation technology for vital sign monitoring in patient monitors, as used in intensive care units or operating rooms. It was developed based on user-centred design [2,10] and cognitive engineering principles [11,12], in order to increase care providers' situation awareness. It does so by improving the exchange of information at the human-machine interface. The technology creates a virtual model of the monitored patient from the monitoring data. The information content is exaggerated but presented realistically. When blood pressure is high, the avatar's body begins to pulse firmly; when oxygen saturation is low, it turns purple, and when brain activity is low, it closes its eyes. This type of display eliminates the need for users to translate the monitor's numbers before interpreting them cognitively, and is better adapted to the strengths of human sensory perception and information processing [13-15]. In several computer-based studies, Visual-Patient-avatar facilitated time-efficient monitoring, increased the users' diagnostic confidence and lowered their perceived workload [13-17]. In addition, VisualPatient-avatar improved the ability to monitor multiple patients simultaneously [18], and was described as intuitive and easy to learn [19-21]. A recent simulation study found quicker recognition of the cause of simulated emergencies when teams used avatar-based monitoring [22]. The previous studies were conducted with the initial prototype design created at the University of Zurich. Although this design was functionally good, designwise, it was very rudimentary and not optimally proportioned. For example, the head was too large and the thorax too small. Since an aesthetically optimised appearance may improve care providers' acceptance and a technology's perceived usability [23], the initial design of Visual-Patient-avatar required adaptions in terms of functionality and aesthetics before its introduction to clinical reality.

This study investigated first impressions of avatar-naive anaesthesia providers regarding the original design of Visual-Patient-avatar. Based on these findings, the avatar was redesigned. The study then explored general acceptance of the new, revised design and understanding of the redesigned visualisations of avatar vital signs in a new group of avatar-naive participants. This study aimed to improve the acceptance and functionality of Visual-Patient-avatar technology through an evidence-based redesign process.

\section{Methods}

The responsible ethics committee reviewed the study protocols and issued declarations of non-responsibility (Business Management System for Ethics Committees numbers Req2020-00059 and Req-2021-00393). All participants provided written informed consent for the anonymous use of their data.

\subsection{Study Design and Participants}

This was an investigator-initiated, prospective, single-centre, mixed qualitative and quantitative study conducted at the University Hospital Zurich in Switzerland, a tertiary 
care facility. This study consisted of three separate parts; a flowchart is provided as Figure 1. The first, a qualitative study, aimed to explore anaesthesia providers' first impressions regarding the original, unrevised design of Visual-Patient-avatar. Structured interviews were conducted with Visual-Patient-avatar-naive subjects. After a qualitative interview analysis, the second study part, the redesign followed, in which the avatar's design was adapted based on the obtained results. The third, final part of the study aimed to quantify general acceptance of the new, revised design, and the level of intuitive recognition of the redesigned vital sign visualisations. The first and third study parts included participants who worked either as resident or staff anaesthesiologists, or as anaesthesia nurses in the designated study centre. Only participants without previous contact with Visual-Patientavatar were included.

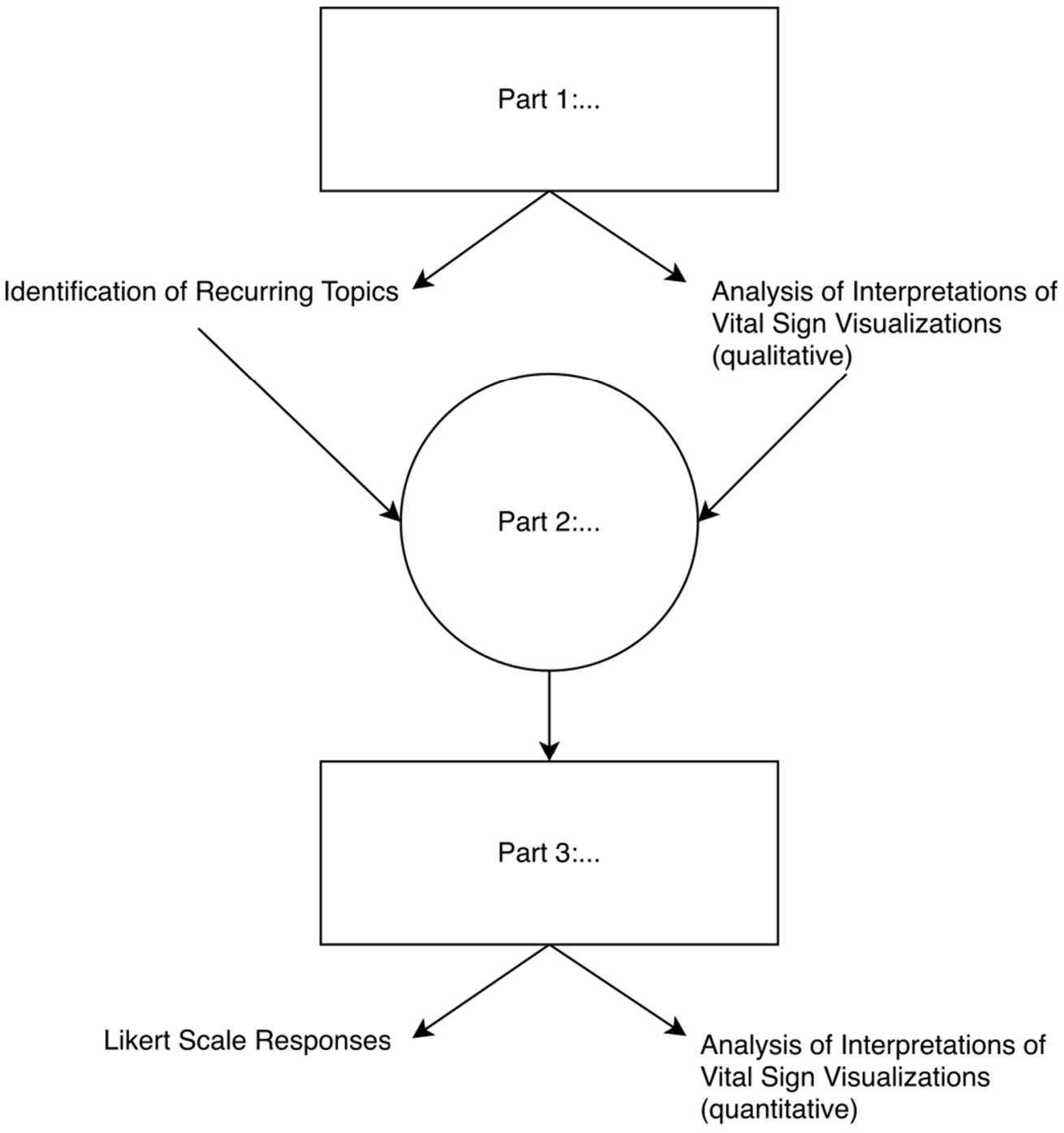

Figure 1. A flowchart outlining the study's design, methodology and parts.

\subsection{The First, Qualitative Study Part}

\subsubsection{Description Qualitative Interview}

For the qualitative first part of the study, four Visual-Patient-avatar scenarios reflecting different patient conditions before or during general anaesthesia were presented. In a quiet and distraction-free environment, participants were instructed to express aloud their feelings, thoughts and ideas while looking at the scenarios. Supplementary Materials File S1 depicts all four scenarios in detail, Supplementary Materials File S2 provides the exact wording of the standardised instructions and Supplementary Materials File S3 provides an 
example video of a participant interview. The scenarios were presented in sequence for one minute each, on a Philips IntelliVue MX500 patient monitor. Participants verbalised their reflections in their native German language, so that no potential language barrier limited their thought processes. Participants were informed that no further information on Visual-Patient-avatar would be provided until after the study session, in order to prevent influencing results. Audio of the structured interviews was recorded using a Sony ICD-UX560 voice recorder (Sony Corp., Minato, Tokyo, Japan). Subsequently, all audio recordings were automatically transcribed using the transcription programme fx4 (Dr. Dresing \& Pehl GmbH, Marburg, Germany). Following the transcription rules listed in Supplementary Materials File S4, the automatically created transcript was revised manually. To qualitatively analyse the transcripts, the template approach [24], a highly structured method for qualitative research, was applied. Major topics with hierarchical subthemes were identified based on word count using Microsoft Word (Microsoft Corporation, Redmond, WA, USA) and free inductive coding based on recurring participants' answers. The resulting template was modified until the coders agreed upon a final version. Figure 2 shows this final coding template, which was then applied to investigate the obtained data. The decision to conduct a think-aloud-like study without training participants on the technology beforehand was based on the belief that unbiased, non-pre-primed thoughts are particularly valuable for identifying the strengths and shortcomings of a technology. This procedure creates a situation like when a person walks into an intensive care unit or operating room and sees the avatar on the screen for the first time. It was considered worthwhile to use the opportunity as long as it would still be possible. Once it is introduced in reality, videos will circulate on the Internet, and care providers will have pre-formed opinions or have learned how to interpret certain visualisations.

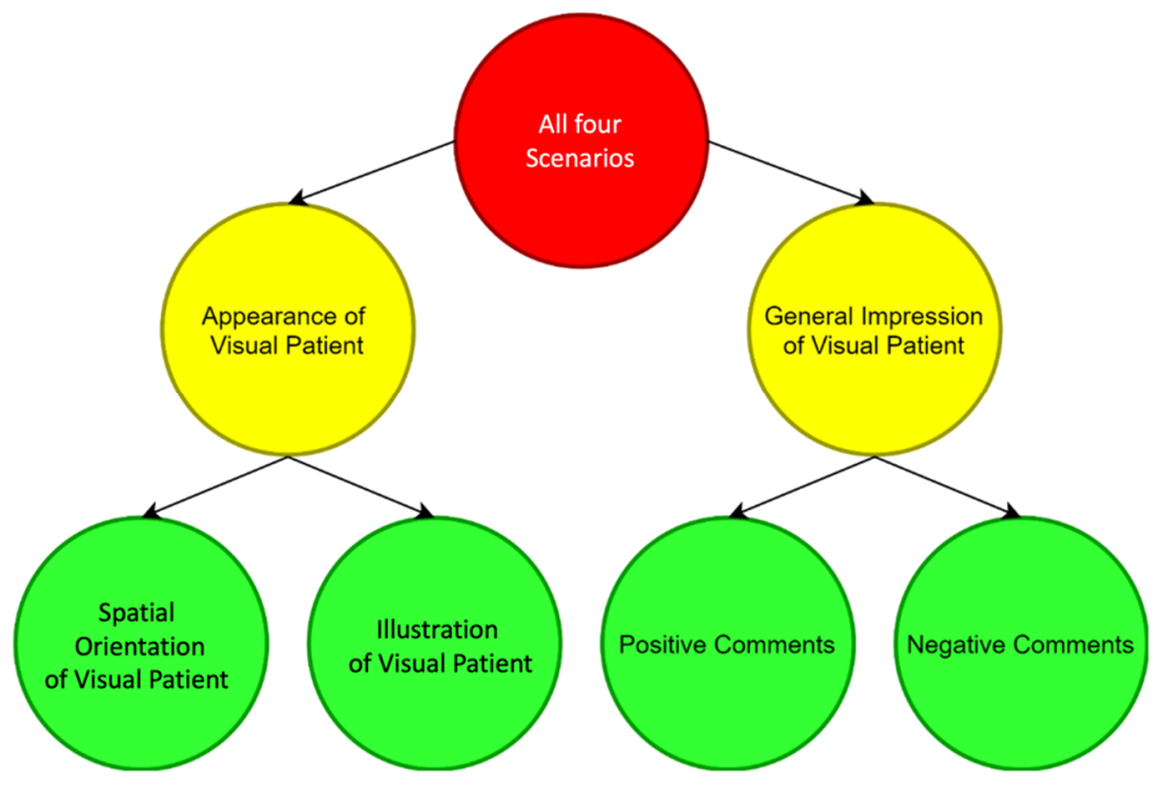

Figure 2. The coding template generated using deductive coding and free inductive coding based on recurring answers in the structured interview transcripts.

\subsubsection{Analysis of Vital Sign Visualisations}

Further transcription analysis focused on the intuitiveness of vital sign visualisations. The transcripts were examined for unsolicited, spontaneous statements about the depicted vital signs. This analysis assessed whether interpretations were, in the intended sense, non-specific (without assuming a possible meaning) or incorrect. This assessment aimed to provide insight into which illustrations may benefit from a redesign for better intuitive understanding. 


\subsection{The Second, Redesign Study Part}

Based on the identified improvement opportunities from the first, qualitative study part, the avatar was redesigned in collaboration with Philips' design and technical specialists between June 2020 and April 2021. This process reviewed the overall appearance of Visual-Patient-avatar, including all vital sign visualisations.

\subsection{The Third, Quantitative Study Part}

\subsubsection{Description of Quantitative Assessment}

In this computer-based study part, the newly revised design was quantitatively analysed with a new group of anaesthesia providers. An iSurvey-based questionnaire (Harvest Your Data, Wellington, New Zealand) running on an iPad (Apple Inc., Cupertino, CA, USA) was used to input data. Again, all data were collected in a quiet, distraction-free setting. In a standardised manner, outlined in Supplementary Materials File S5, the basic concept of Visual-Patient-avatar was explained without clarifying each vital sign in detail. The participants were asked to answer four questions based on themes identified in the previous first, qualitative study part, and during the redesign process. The first question asked about the preferred orientation of Visual-Patient-avatar on the monitor screen (answer options: upright, $45^{\circ}$ clockwise rotation, $135^{\circ}$ clockwise rotation with head down). The second question used a five-point Likert scale from disagree to agree to assess the level of consent with the statement that the avatar's revised skin colour reflects all of humanity. The third question asked about the preferred size of Visual-Patient-avatar using a split-screen mode, where conventional and avatar monitoring modalities are displayed side-by-side on the screen (answer options: $50 \%, 25 \%$ or $12.5 \%$ of the screen area). The fourth question used a five-point Likert scale from disagree to agree to evaluate whether the avatar's appearance looks professional enough for medical use. The answers are presented as proportions and percentages. For the skin colour and design professionalism questions, the Wilcoxon signed-rank test was used to determine whether the sample medians differed significantly from neutral. A $p$-value of $<0.05$ was considered to be statistically significant. Microsoft Excel and Prism 8.4.1 (GraphPad Software, San Diego, CA, USA) were used to display and analyse the quantitative data.

\subsubsection{Analysis of the Vital Sign Visualisations}

The participants were also asked to identify twenty vital sign visualisations in a randomised order using videos with the revised design. Supplementary Materials File S6 provides an example video. These videos were structured in such a way that the avatar was first shown in a normal vital sign state, and then with a single changed vital parameter. Using the iSurvey questionnaire, the participants indicated which vital sign visualisation they thought had changed. The results of these questions are presented as proportions and percentages. For the analysis, we used Microsoft Excel and Prism 8.4.1.

\section{Results}

\subsection{Study and Participant Characteristics}

Between 4 May and 28 May 2020, 51 interviews with 51 participants were conducted for the first, qualitative study part, all of which were transcribed and analysed. Between 12 April and 20 April 2021, 30 new participants were recruited for the third, quantitative study part. $99 \%$ (718 of 720 ) of the questions were answered, and all questionnaires (30 of 30) analysed. Table 1 provides an overview of the study characteristics. 
Table 1. Study and participant characteristics.

\begin{tabular}{|c|c|c|}
\hline & First, Qualitative Study Part & Third, Quantitative Study Part \\
\hline Study Design & $\begin{array}{l}\text { Interview-Based } \\
\text { Assessment }\end{array}$ & Computer-Based Survey \\
\hline $\begin{array}{l}\text { Number of participants; } N 1 \\
\text { and N2, respectively. }\end{array}$ & 51 & 30 \\
\hline $\begin{array}{c}\text { Gender of participants; } n \\
\text { female of } N 1(\%) \text { and n } \\
\text { female of } N 2(\%), \\
\text { respectively }\end{array}$ & 31 of $51(61 \%)$ & 18 of $30(60 \%)$ \\
\hline $\begin{array}{l}\text { Number of physicians; } \mathrm{n} \text { of } \\
\text { N1 (\%) and n of } \mathrm{N} 2(\%) \\
\text { respectively. }\end{array}$ & 28 of $51(55 \%)$ & 24 of $30(80 \%)$ \\
\hline $\begin{array}{l}\text { Nurse anaesthetists; } \mathrm{n} \text { of } \mathrm{N1} \\
\text { (\%) and n of } \mathrm{N} 2(\%), \\
\text { respectively. }\end{array}$ & 23 of $51(45 \%)$ & 6 of $30(20 \%)$ \\
\hline $\begin{array}{l}\text { Anaesthesia experience in } \\
\text { years; median (IQR [range]) }\end{array}$ & $5.5(2.0-7.5[1.0-36.0])$ & $2.0(1.0-3.0[1.0-30.0])$ \\
\hline
\end{tabular}

\subsection{The First, Qualitative Study Part}

\subsubsection{Interview Analysis}

The Consolidated Criteria for Reporting Qualitative Research (COREQ) [25] and the Standards for Reporting Qualitative Research (SRQR) [26] were followed in this study. Two main themes with two subthemes each were identified, and participant statements from the transcribed interviews were grouped accordingly. Figure 2 depicts the generated coding template. Table 2 illustrates the transcripts' qualitative analysis using the coding template.

Table 2. Main themes/subthemes with statement count and exemplary responses. $N=51$ participants.

\begin{tabular}{|c|c|c|}
\hline Main Theme & Subtheme & Examples \\
\hline \multirow[t]{2}{*}{$\begin{array}{l}\text { Appearance of Visual- } \\
\text { Patient-avatar } \\
\text { ( } 33 \text { statements made } \\
\text { by } 28 \text { of } 51[55 \%] \\
\text { participants) }\end{array}$} & $\begin{array}{l}\text { Illustration of Visual- } \\
\text { Patient-avatar } \\
\text { ( } 22 \text { statements made } \\
\text { by } 21 \text { of } 51 \text { [ } 43 \%] \\
\text { participants) }\end{array}$ & $\begin{array}{l}\text { Participant \#3: Looks a bit childish, very } \\
\text { simple design. } \\
\text { Participant \#6: Still seems somewhat artificial. } \\
\text { Participant \#10: I feel like I'm in kindergarten } \\
\text { at the moment when I see the figure. } \\
\text { Participant \#14: It has a very big head in } \\
\text { contrast to the rest of the body. } \\
\text { Participant \#16: Is not very truthfully } \\
\text { portrayed. } \\
\text { Participant \#21: A bit bare, dull. }\end{array}$ \\
\hline & $\begin{array}{l}\text { Spatial orientation of } \\
\text { Visual-Patient- } \\
\text { avatar } \\
\text { (11 statements made } \\
\text { by } 11 \text { of } 51[22 \%] \\
\text { participants) }\end{array}$ & $\begin{array}{l}\text { Participant \#17: It's a bit unusual to see it like } \\
\text { that. Um especially because it's upside down. } \\
\text { Participant \#28: The position confuses me a bit. } \\
\text { Participant \#30: A patient who is lying upside } \\
\text { down. } \\
\text { Participant \#32: I honestly have trouble with } \\
\text { him lying with his head down. }\end{array}$ \\
\hline $\begin{array}{l}\text { General impression of } \\
\text { Visual-Patient-avatar } \\
\text { ( } 26 \text { statements made } \\
\text { by } 23 \text { of } 51[45 \%] \\
\text { participants) }\end{array}$ & $\begin{array}{l}\text { Positive comments } \\
\text { (14 statements made } \\
\text { by } 14 \text { of } 51[28 \%] \\
\text { participants) }\end{array}$ & $\begin{array}{l}\text { Participant \#4: Yes, looks fun. } \\
\text { Participant \#6: Really funny. Something } \\
\text { positive. } \\
\text { Participant \#7: Quite funny, yes. } \\
\text { Participant \#14: Okay, it is cute. } \\
\text { Participant \#33: At first glance, probably rather } \\
\text { a funny figure. }\end{array}$ \\
\hline
\end{tabular}


Table 2. Cont.

\begin{tabular}{|c|c|c|}
\hline Main Theme & Subtheme & Examples \\
\hline & $\begin{array}{l}\text { Negative comments } \\
\text { (12 statements made } \\
\text { by } 12 \text { of } 51[24 \%] \\
\text { participants) }\end{array}$ & $\begin{array}{l}\text { Participant \#21: So, I find the picture quite } \\
\text { unclear. I do not know what it wants to tell me. } \\
\text { Participant \#27: At the moment, I could still } \\
\text { imagine having trouble with that, if that's all I } \\
\text { see. } \\
\text { Participant \#32: I am a bit irritated by the } \\
\text { presentation. } \\
\text { Participant \#36: No idea. I do not know what } \\
\text { to do with it. }\end{array}$ \\
\hline
\end{tabular}

\subsubsection{Vital Sign Visualisation Analysis}

In the interview transcripts, 756 statements mentioning vital signs were identified. Supplementary Materials File S7 breaks down the comments by vital sign and scenario. Supplementary Materials File S8 contains the complete dataset for the vital sign analysis. The most frequently correctly interpreted vital sign was body temperature, with $76 \%$ (38 of 51) accurate statements. All other vital signs were rated correctly in less than half of the statements. Central venous pressure was the vital sign with the fewest accurate statements (5 of $51[10 \%]$ statements).

\subsection{The Second, Redesign Study Part}

Based on the results of the first, qualitative analysis, Visual-Patient-avatar was redesigned from July 2020 until the end of March 2021. Since the participants often criticised the avatar's shape, we modified its body proportions to resemble the human shape more closely. Among others, the thorax area was enlarged to allow better visibility of the illustrations in the thoracic area. As participants also commented on the avatars' original spatial orientation $\left(135^{\circ}\right.$ clockwise rotated with the head down), two additional positions were created with an upright avatar and a $45^{\circ}$ clockwise rotated position. Furthermore, several vital sign visualisations were redesigned to increase their intuitive recognisability. This was accomplished by improving the anatomical correctness of the representations. For example, the illustration of central venous pressure, which initially was frequently misrecognised, was designed more anatomical. Finally, the avatar's skin colour had to be adapted from a Caucasian skin tone to a darker complexion to cover a larger part of global population. Figure 3 shows Visual-Patient-avatar before and after the redesign.

\subsection{The Third, Quantitative Study Part \\ 3.4.1. Survey Analysis}

The general questions had a $100 \%$ response rate. Concerning the spatial orientation, 28 of $30(93 \%)$ participants preferred the upright or nearly upright orientation. When asked about the avatars' skin colour, 16 of 30 (53\%) participants rather agreed or agreed to the statement that the colour was a good choice. Using the Wilcoxon signed-rank test, these answers differed significantly from neutral, with $p=0.008$. Regarding the split-screen monitor, 28 of 30 (93\%) participants favoured a Visual-Patient-avatar size of $25 \%$ of the monitor or more. Twenty of 30 (67\%) participants rather agreed or agreed to the statement that the redesign looks professional enough for medical use. The Wilcoxon signed-rank test showed that mentioned answers differed significantly from neutral, $p<0.001$. Figure 4 shows a detailed overview of the quantitative survey results. 

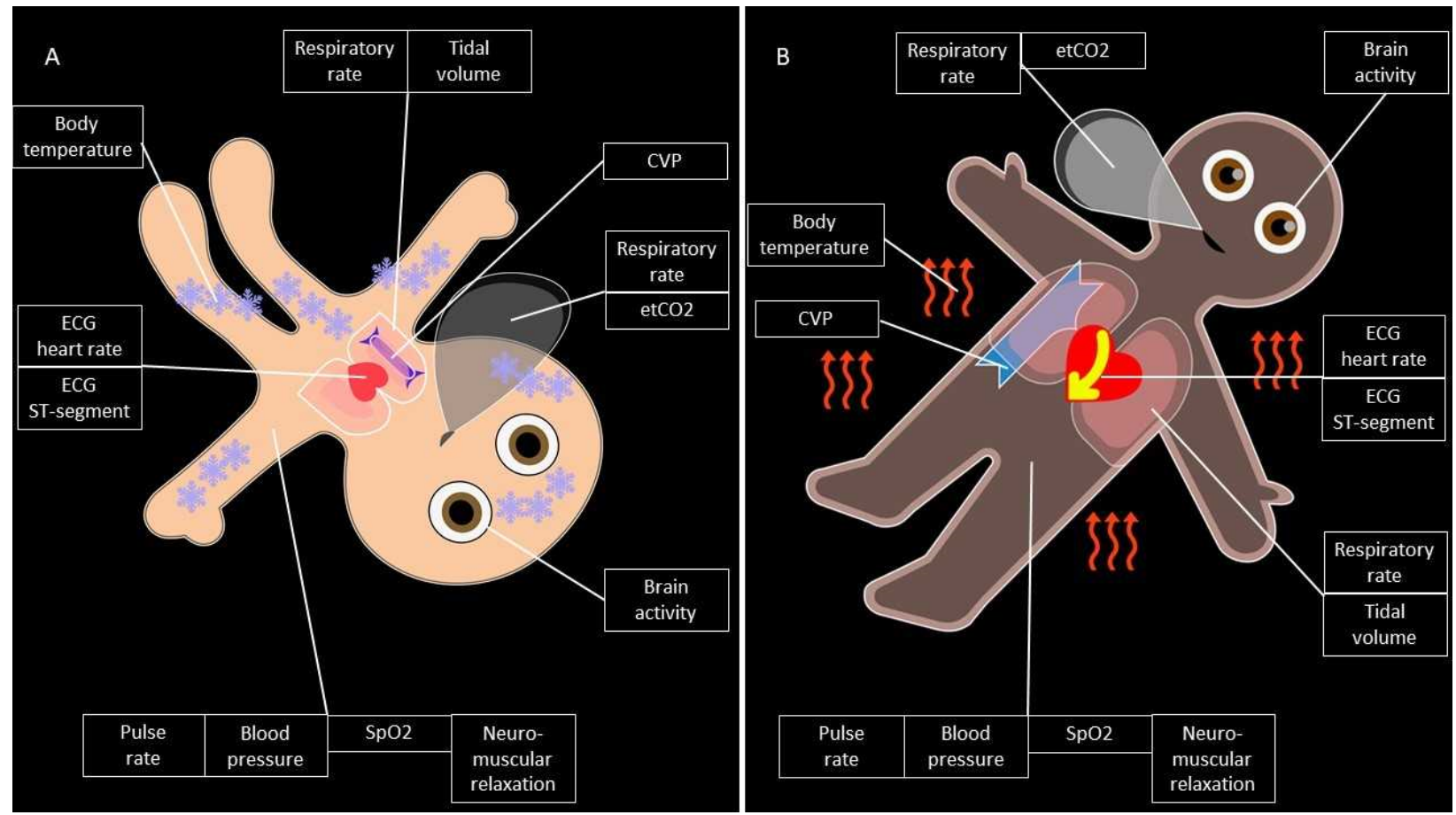

Figure 3. The Visual-Patient-avatar before (A) and after (B) the redesign process. CVP: central venous pressure, ECG: electrocardiogram, etCO2: end-tidal carbon dioxide, $\mathrm{SpO}$ : peripheral oxygen saturation. When the body temperature is within the normal range, neither ice crystals nor heatwaves are visible.
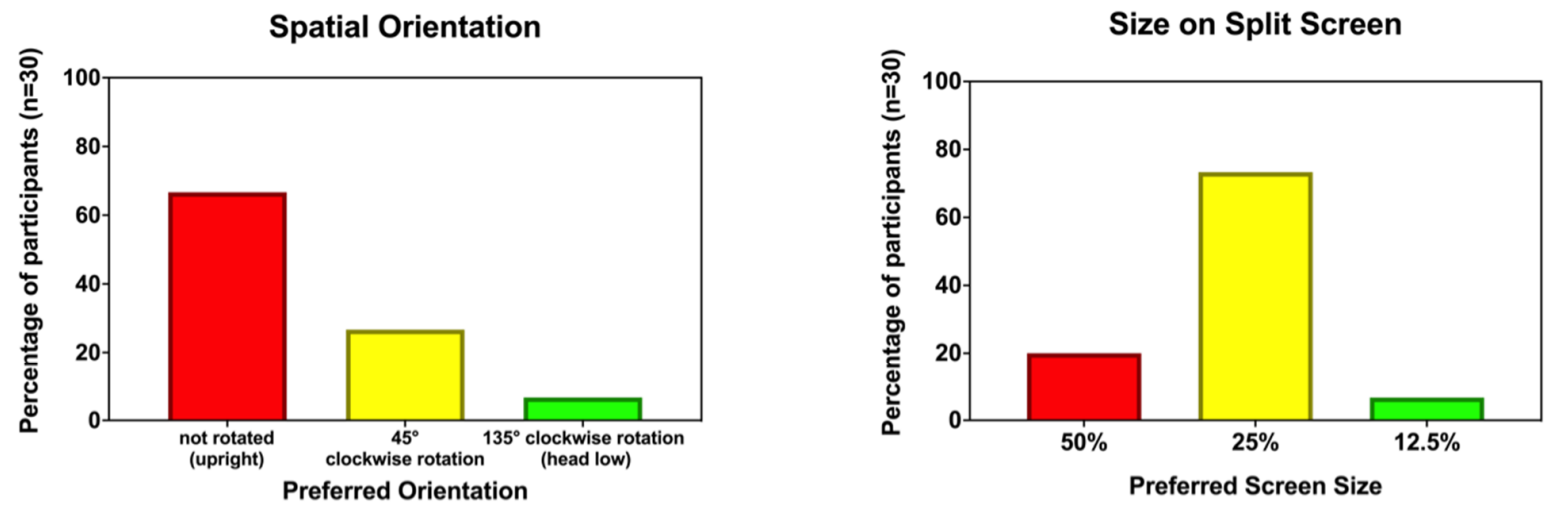

Professionalism
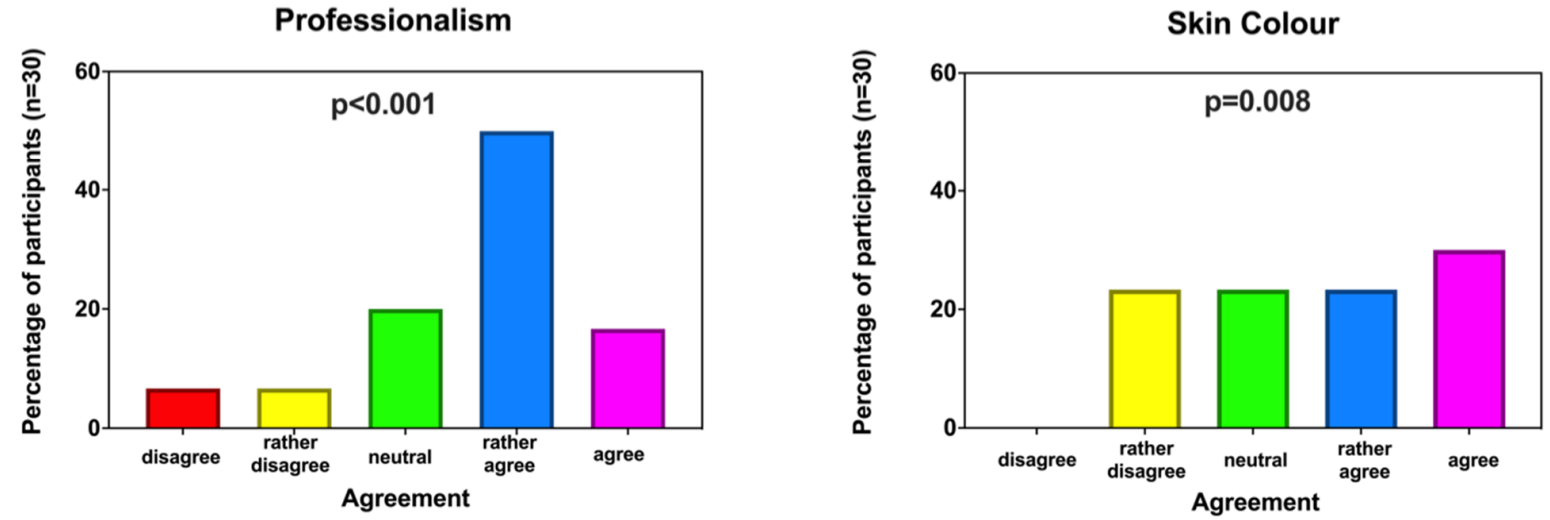

Figure 4. Responses to the general questions in the third, quantitative study part. The $p$-value indicates statistical difference from neutral (with Wilcoxon signed-rank test). 


\subsubsection{Vital Signs Visualisation Analysis}

Figure 5 shows a detailed analysis of intuitive interpretation accuracy concerning vital sign visualisations. In total, the participants rated 600 visualisations. Two of $600(0.3 \%)$ answers were missing due to app-based technical issues and 435 of 600 (73\%) answers were correct. Twenty-three of $30(77 \%)$ participants misinterpreted the two vital sign visualisations, depicting low blood pressure and ST-segment deviations. The anaesthesia personnel intuitively recognised the other 18 vital sign changes with accuracies of $57 \%$ or higher. Central venous pressure, which had hardly been recognised in the first study part, performed well in the quantitative analysis after its redesign. Low central venous pressure was interpreted correctly in 26 of $30(87 \%)$ answers. High central venous pressure was identified correctly in 27 of $30(90 \%)$ answers.

\section{Correct Interpretation of Vital Sign Visualisations}

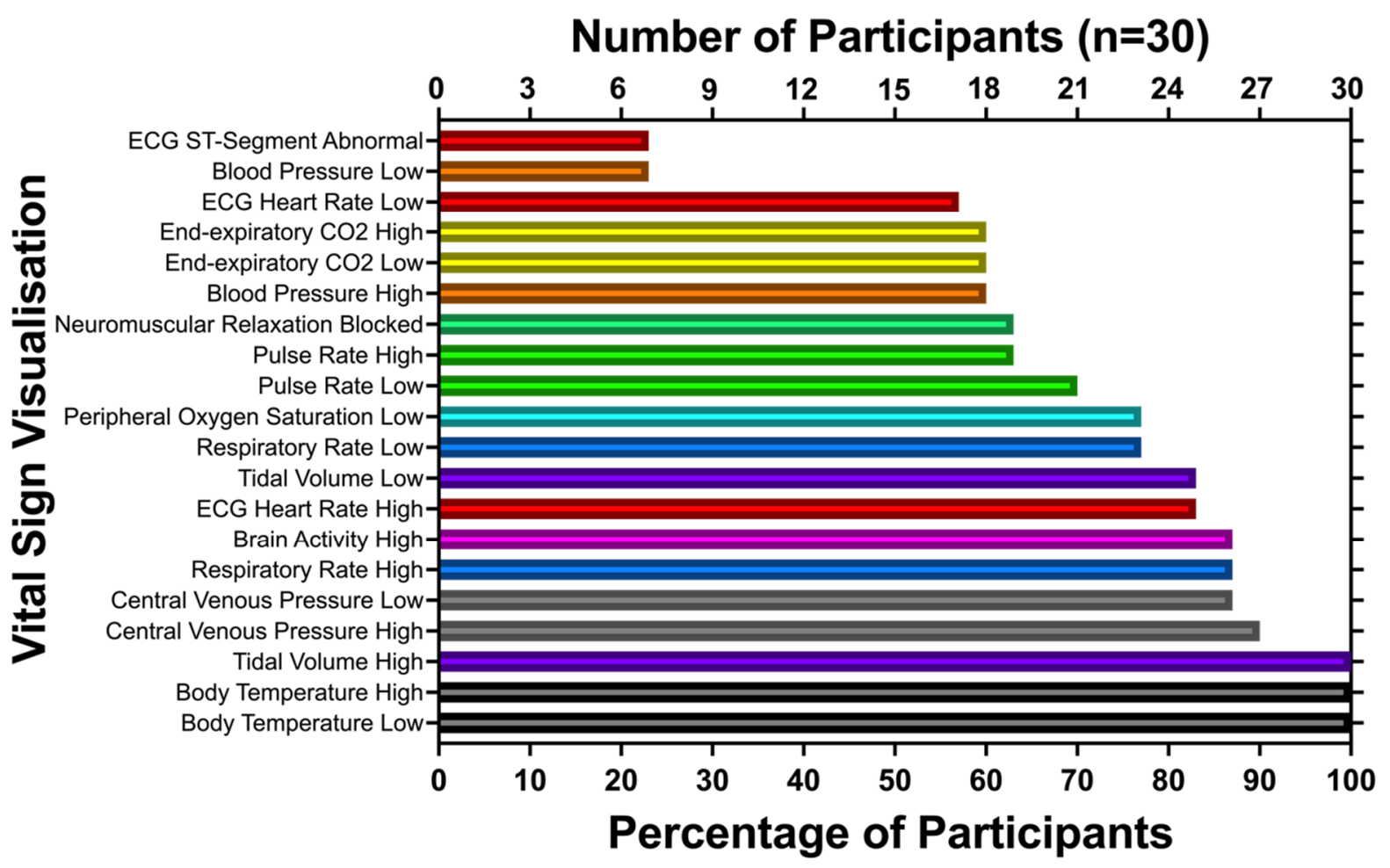

Figure 5. Intuitive interpretation accuracy per vital sign deviation. The correct interpretations are given as answer count and percentages. A maximum of 30 out of $30(100 \%)$ interpretations per vital signs were correct.

\section{Discussion}

This mixed-method study's main objective was to render the redesign of Visual-Patientavatar a scientific process. Based on the qualitative results of the structured interviews, the avatar's shape, spatial orientation, skin colour and several vital sign visualisations were redesigned. After the redesign process, a quantitative assessment found high approval regarding the modifications.

The interview analysis succeeded in revealing design aspects that participants recurrently identified as disturbing or unclear. Redesigning these issues was considered critical for ensuring the acceptance and usability of Visual-Patient-avatar. The original version depicted the avatar in a comic-style design with a spatial perspective that turned the avatar upside down to mirror the anaesthesiologist's usual perspective at the patient's head. As participants repeatedly commented on these aspects, two new versions with the avatar's head pointed upwards were designed. The main design focus was on anatomically correct 
representations with simplified body parts while maintaining realistic proportions. The intention behind the simplified, cartoon-style presentation was to avoid more complex design elements that would potentially distract from essential information and produce a mental workload to interpret its meaning. A guiding principle of user-centred design is to narrow down presentation to the most critical information based on users' objectives [2].

The share of Visual-Patient-avatar on the monitor is a relevant aspect for its clinical introduction. Seventy-three percent of participants preferred $25 \%$ of the monitor screen area. This proportion seems in line with other situation awareness-oriented visualisations in medical devices, such as the dynamic lung product available in Hamilton ventilators (Hamilton Medical AG, Bonaduz, Switzerland) [27,28].

One of the main design modifications concerned the avatar's skin colour. As VisualPatient-avatar technology is intended for global use, it became clear that a revision of skin tone was necessary. The quantitative survey showed that no one completely disagreed and 16 of 30 (53\%) participants rather agreed or agreed the redesign was a good compromise for the representation of all humanity. Supported by this result, this new skin colour was kept for the clinical rollout of Visual-Patient-avatar. This modification is significant, considering global efforts to achieve equality for all ethnic groups. Selecting one colour for everyone seemed more desirable than introducing different colours and having to switch between them.

In the original design, some vital sign visualisations proved difficult to interpret without explanations. According to the quantitative assessment of the revised design, the more anatomically correct vital sign modifications led to a $73 \%$ probability to intuitively recognise the visualisations in their intended sense, where 'intuitively' means without any teaching or training whatsoever. Other researchers evaluating user-centred interfaces also observed that anatomically accurate representations promote intuitive understanding [10]. In medical technology, the question regarding the relevance of intuitive understanding often arises [29,30], as some training can usually be expected. In the past, however, research has attributed counterintuitive design as a factor responsible for the failure of medical visualisation technologies [7], which is why this aspect remains relevant. According to the definition of Blackler and colleagues, intuition is a type of cognitive processing that is often, but not always, non-conscious, and utilises stored experiential knowledge [31]. In previous studies, anaesthesia and intensive care personnel quickly understood the avatar visualisations after obtaining a short instructional video [14-16,18,19,22,32]. Even after the redesign, however, there remain ambiguities in the intuitive assessment of some vital signs, especially ST-segment deviations and low blood pressure. Therefore, despite its simple design features geared at intuitive understanding, some Visual-Patient-avatar teaching should be provided before clinical use. The level of intuitive understanding found in this study suggests that the technology succeeds in reaching the goal of being a tool designed to promote situation awareness, which is to transfer the relevant information as quickly as possible, with the lowest possible cognitive stress, while easing adoption and use [2]. The clinical importance of increasing situation awareness is evident in the relationship between situation awareness breakdowns and adverse events [3-6].

\section{Limitations and Strengths}

This study had several limitations. First, this was a monocentric central European study conducted in the hospital where Visual-Patient-avatar was invented. Opinions may differ elsewhere in the world. Second, even though the study was conducted according to the COREQ [25] and SRQR [26] criteria, qualitative research inherently includes subjective judgment in data collection and analysis. As both the study team and the participants consisted of anaesthesia personnel, this shared background may have influenced the subjective assessments. Furthermore, the measured types of intuitive vital sign interpretation in the first, qualitative and third, quantitative part are not the same, and thus a direct comparison is not possible. In the first, qualitative study part, the participants were intentionally not instructed to interpret the vital signs, in order to avoid investigator-induced mental precon- 
ceptions; this meant that respondents did not always attempt to interpret each visualisation. Thus, this part of the study enabled us to get an impression of the participants' unconscious intuitions. According to the definition of intuition of Blackler and colleagues [31], in the third, quantitative study part, conscious intuition was measured, as the participants were explicitly instructed to interpret the visualisations.

The qualitative interview approach is not just a limitation. It is one of the study's strengths, as it allowed us to obtain unbiased and broad feedback, while the survey enabled us to weigh relevant aspects of the identified themes quantitatively. Another strength is the balanced sample in terms of gender and occupation in both study parts. These facts and the high response rate reduced a possible sample bias.

\section{Conclusions}

Using an innovative study approach allowed us to improve and fine-tune the design and concept of Visual-Patient-avatar. Intuitiveness and perceived professionalism achieved a good level in the new, more anatomically correct design. A head-up rotated version with a screen size of $25 \%$ seemed to be preferred by anaesthesia personnel, and may therefore serve as the default version. Future studies may further tweak the few visualisations that still had low intuitiveness after the redesign, and may analyse the effects of giving them special consideration in instructional material. Future studies must continue to evaluate the performance of the concept and design in real-life clinical use. These studies should incorporate feedback from a global population, and continue to refine vital sign illustrations with the goal of achieving an avatar product that maintains its functionality, and is accessible for the broadest possible group of professional healthcare providers and everyone who may come into contact with it, e.g., patients and their family members.

Supplementary Materials: The following supporting information can be downloaded at: https: / / www.mdpi.com/article/10.3390/diagnostics12020555/s1. Supplementary Materials File S1 graphically depicts all four scenarios in more detail, which were shown to participants in the first, qualitative study part for the structured interviews. Supplementary Materials File S2 provides the exact wording of the standardised instructions of the first, qualitative study part, which were given to all 51 participants. Supplementary Materials File S3 provides an example video of a participant interview of the first, qualitative study part. Link: https://1drv.ms/v/s!AjkumfX_cNxMi45b7ZW2 rZ92LJboUw? $=$ rKdRIM. Supplementary Materials File S4 includes the transcription rules, which were used to transcript the interviews of the first, qualitative study part. Supplementary Materials File S5 provides the exact wording of the standardised instructions of the third, quantitative study part, which were given to all 30 participants. Supplementary Materials File S6 contains a Visual-Patient-avatar vital sign interpretation video of the third, quantitative study part. Link: https:/ /1drv.ms/v/s!AjkumfX_cNxMi45Y3Lk2iMpb4j-L2g?e=I5E2Wa. Supplementary Materials File S7 lists the vital sign identifications in the interview-transcripts of the first, qualitative study part. Supplementary Materials File S8 is a complete dataset for the vital sign identification analysis.

Author Contributions: D.J.W., L.B., C.B.N., S.S. and D.W.T. helped to design the study; D.J.W., L.B., C.B.N., S.S. and D.W.T. helped to collect the data; D.J.W., L.B., S.S. and D.W.T. helped to analyse the data; and D.J.W., L.B., C.B.N., S.S., T.R.R., D.R.S. and D.W.T. helped to write the manuscript and approved the final version. All authors have read and agreed to the published version of the manuscript.

Funding: The Institute of Anaesthesiology at the University Hospital Zurich in Switzerland funded this study.

Institutional Review Board Statement: The responsible ethics committee issued declarations of non-responsibility. Business Management System for Ethics Committees numbers Req-2020-00059 and Req-2021-00393).

Informed Consent Statement: Informed consent was obtained from all subjects involved in the study.

Data Availability Statement: The original data are available in the Supplementary Materials. 
Acknowledgments: The authors are thankful to the study participants for their time and effort. Furthermore, the authors thank Dressing \& Pehl GmbH (Marburg, Germany) for the free transcription time provided in their $\mathrm{fx} 4$ transcription programme, and Thomas Campbell-James for his language input.

Conflicts of Interest: D.W.T. and C.B.N. are designated inventors of Visual-Patient-avatar technology, for which the University of Zurich holds various patents and trademarks. There are cooperation and licensing agreements with Philips Medizin Systeme Böblingen $\mathrm{GmbH}$, Böblingen, Germany; Koninklijke Philips NV, Amsterdam, the Netherlands; Philips Research/Philips Electronics Nederland BV, Eindhoven, the Netherlands, and Philips North America, Cambridge, MA, USA. Under these agreements, D.W.T. receives research funding, D.W.T. and C.B.N. receive travel support and may receive royalties. D.W.T., C.B.N. and D.R.S. are designated inventors of Visual Clot technology, for which the University of Zurich holds various patents and trademarks. The University of Zurich signed a letter of intent for a cooperation and licensing agreement with Instrumentation Laboratory Company/Werfen Corporation, Bedford, MA, USA and Barcelona, Spain. Under this and future agreements, they may receive royalties. D.W.T., C.B.N. and D.R.S. received travel support for consulting Instrumentation Laboratory, Bedford, MA, USA. DRS's academic department receives grant support from the Swiss National Science Foundation, Berne, Switzerland, the Swiss Society of Anaesthesiology and Reanimation (SGAR), Berne, Switzerland, the Swiss Foundation for Anaesthesia Research, Zurich, Switzerland, Vifor SA, Villars-sur-Glâne, Switzerland and Vifor (International) AG, St. Gallen, Switzerland. DRS is co-chair of the ABC-Trauma Faculty, sponsored by unrestricted educational grants from Novo Nordisk Health Care AG, Zurich, Switzerland, CSL Behring GmbH, Marburg, Germany, LFB Biomédicaments, Courtaboeuf Cedex, France and Octapharma AG, Lachen, Switzerland. DRS received honoraria/travel support for consulting or lecturing from: Danube University of Krems, Austria, US Department of Defense, Washington, USA, European Society of Anaesthesiology, Brussels, BE, Korean Society for Patient Blood Management, Seoul, Korea, Korean Society of Anesthesiologists, Seoul, Korea, Network for the Advancement of Patient Blood Management, Haemostasis and Thrombosis, Paris, France, Baxalta Switzerland AG, Volketswil, Switzerland, Bayer AG, Zürich, Switzerland, B. Braun Melsungen AG, Melsungen, Germany, Boehringer Ingelheim $\mathrm{GmbH}$, Basel, Switzerland, Bristol-Myers-Squibb, Rueil-Malmaison Cedex, France and Baar, Switzerland, CSL Behring GmbH, Hattersheim am Main, Germany and Berne, Switzerland, Celgene International II Sàrl, Couvet, Switzerland, Daiichi Sankyo AG, Thalwil, Switzerland, Haemonetics, Braintree, MA, USA, Instrumentation Laboratory (Werfen), Bedford, MA, USA, LFB Biomédicaments, Courtaboeuf Cedex, France, Merck Sharp \& Dohme, Kenilworth, New Jersey, USA, Novo Nordisk Health Care AG, Zurich, Switzerland, PAION Deutschland GmbH, Aachen, Germany, Pharmacosmos A/S, Holbaek, Denmark, Pfizer AG, Zürich, Switzerland, Pierre Fabre Pharma, Alschwil, Switzerland, Portola Schweiz GmbH, Aarau, Switzerland, Roche Diagnostics International Ltd., Reinach, Switzerland, Sarstedt AG \& Co., Sevelen, Switzerland and Nümbrecht, Germany, Shire Switzerland GmbH, Zug, Switzerland, Tem International GmbH, Munich, Germany, Vifor Pharma, Munich, Germany, Neuilly sur Seine, France and Villars-sur-Glâne, Switzerland, Vifor (International) AG, St. Gallen, Switzerland, Zuellig Pharma Holdings, Singapore, Singapore. The other authors do not have any conflicts of interests.

\section{References}

1. World Health Organization. Guidelines for Safe Surgery 2009: Safe Surgery Saves Lives; WHO: Geneva, Switzerland, 2009.

2. Endsley, M.R.; Jones, D.G. Designing for Situation Awareness: An Approach to User-Centered Design, 2nd ed.; CRC Press: Boca Raton, FL, USA, 2011.

3. Schulz, C.M.; Endsley, M.R.; Kochs, E.F.; Gelb, A.W.; Wagner, K.J. Situation awareness in anesthesia: Concept and research. Anesthesiology 2013, 118, 729-742. [CrossRef] [PubMed]

4. Fioratou, E.; Flin, R.; Glavin, R.; Patey, R. Beyond monitoring: Distributed situation awareness in anaesthesia. Br. J. Anaesth. 2010, 105, 83-90. [CrossRef] [PubMed]

5. Schulz, C.M.; Burden, A.; Posner, K.L.; Mincer, S.L.; Steadman, R.; Wagner, K.J.; Domino, K.B. Frequency and Type of Situational Awareness Errors Contributing to Death and Brain Damage: A Closed Claims Analysis. Anesthesiology 2017, 127, 326-337. [CrossRef] [PubMed]

6. Schulz, C.M.; Krautheim, V.; Hackemann, A.; Kreuzer, M.; Kochs, E.F.; Wagner, K.J. Situation awareness errors in anesthesia and critical care in 200 cases of a critical incident reporting system. BMC Anesthesiol. 2016, 16, 4. [CrossRef]

7. Drews, F.A.; Westenskow, D.R. The right picture is worth a thousand numbers: Data displays in anesthesia. Hum. Factors 2006, 48, 59-71. [CrossRef] 
8. Drews, F.A. Patient monitors in critical care: Lessons for improvement. In Advances in Patient Safety: New Directions and Alternative Approaches (Vol. 3: Performance and Tools); Agency for Healthcare Research and Quality (US): Rockville, MD, USA, 2008.

9. Rayner, K. Eye movements in reading and information processing: 20 Years of research. Psychol. Bull. 1998, 124, 372-422 [CrossRef]

10. Wachter, S.B.; Agutter, J.; Syroid, N.; Drews, F.; Weinger, M.B.; Westenskow, D. The employment of an iterative design process to develop a pulmonary graphical display. J. Am. Med. Inform. Assoc. 2003, 10, 363-372. [CrossRef]

11. Wittgenstein, L. Tractatus Logico-Philosophicus; Routledge \& Kegan Paul: London, UK, 1922.

12. Degani, A.; Jorgensen, C.; Iverson, D.; Shafto, M.; Olson, L. On Organization of Information: Approach and Early Work; NASA Ames Research Center: Moffett Field, CA, USA, 2009.

13. Tscholl, D.W.; Rossler, J.; Handschin, L.; Seifert, B.; Spahn, D.R.; Nothiger, C.B. The Mechanisms Responsible for Improved Information Transfer in Avatar-Based Patient Monitoring: Multicenter Comparative Eye-Tracking Study. J. Med. Internet Res. 2020, 22, e15070. [CrossRef]

14. Tscholl, D.W.; Handschin, L.; Neubauer, P.; Weiss, M.; Seifert, B.; Spahn, D.R.; Noethiger, C.B. Using an animated patient avatar to improve perception of vital sign information by anaesthesia professionals. Br. J. Anaesth. 2018, 121, 662-671. [CrossRef]

15. Tscholl, D.W.; Rössler, J.; Said, S.; Kaserer, A.; Spahn, D.R.; Nöthiger, C.B. Situation Awareness-Oriented Patient Monitoring with Visual Patient Technology: A Qualitative Review of the Primary Research. Sensors 2020, 20, 2112. [CrossRef]

16. Pfarr, J.; Ganter, M.T.; Spahn, D.R.; Noethiger, C.B.; Tscholl, D.W. Effects of a standardized distraction on caregivers' perceptive performance with avatar-based and conventional patient monitoring: A multicenter comparative study. J. Clin. Monit. Comput. 2020, 34, 1369-1378. [CrossRef] [PubMed]

17. Said, S.; Gozdzik, M.; Roche, T.R.; Braun, J.; Rössler, J.; Kaserer, A.; Spahn, D.R.; Nöthiger, C.B.; Tscholl, D.W. Validation of the Raw National Aeronautics and Space Administration Task Load Index (NASA-TLX) Questionnaire to Assess Perceived Workload in Patient Monitoring Tasks: Pooled Analysis Study Using Mixed Models. J. Med. Internet Res. 2020, 22, e19472. [CrossRef] [PubMed]

18. Garot, O.; Rössler, J.; Pfarr, J.; Ganter, M.T.; Spahn, D.R.; Nöthiger, C.B.; Tscholl, D.W. Avatar-based versus conventional vital sign display in a central monitor for monitoring multiple patients: A multicenter computer-based laboratory study. BMC Med. Inform. Decis. Mak. 2020, 20, 26. [CrossRef] [PubMed]

19. Rössler, J.; Kaserer, A.; Albiez, B.; Braun, J.; Breckwoldt, J.; Spahn, D.R.; Nöthiger, C.B.; Tscholl, D.W. Comparing Classroom Instruction to Individual Instruction as an Approach to Teach Avatar-Based Patient Monitoring With Visual Patient: Simulation Study. JMIR Med. Educ. 2020, 6, e17922. [CrossRef]

20. Tscholl, D.W.; Weiss, M.; Handschin, L.; Spahn, D.R.; Nöthiger, C.B. User perceptions of avatar-based patient monitoring: A mixed qualitative and quantitative study. BMC Anesthesiol. 2018, 18, 188. [CrossRef]

21. Akbas, S.; Said, S.; Roche, T.R.; Nöthiger, C.B.; Spahn, D.R.; Tscholl, D.W.; Bergauer, L. User perceptions of different monitor modalities during high-fidelity simulation: Visual-Patient-avatar; Split Screen and Conventional-A semiquantitative analysis. JMIR Hum. Factors 2022. [CrossRef]

22. Roche, T.R.; Said, S.; Braun, J.; Maas, E.J.C.; Machado, C.; Grande, B.; Kolbe, M.; Spahn, D.R.; Nöthiger, C.B.; Tscholl, D.W. Avatar-based patient monitoring in critical anaesthesia events: A randomised high-fidelity simulation study. Br. J. Anaesth. 2021, 126, 1046-1054. [CrossRef]

23. Tractinsky, N.; Katz, A.S.; Ikar, D. What is beautiful is usable. Interact. Comput. 2000, 13, 127-145. [CrossRef]

24. Brooks, J.; McCluskey, S.; Turley, E.; King, N. The utility of template analysis in qualitative psychology research. Qual. Res. Psychol. 2015, 12, 202-222. [CrossRef]

25. Tong, A.; Sainsbury, P.; Craig, J. Consolidated criteria for reporting qualitative research (COREQ): A 32-item checklist for interviews and focus groups. Int. J. Qual. Health Care 2007, 19, 349-357. [CrossRef]

26. O'Brien, B.C.; Harris, I.B.; Beckman, T.J.; Reed, D.A.; Cook, D.A. Standards for reporting qualitative research: A synthesis of recommendations. Acad. Med. 2014, 89, 1245-1251. [CrossRef] [PubMed]

27. Branson, R.D.; Johannigman, J.A. Innovations in mechanical ventilation. Respir. Care 2009, 54, 933-947. [CrossRef] [PubMed]

28. Ventilation Cockpit User Interface; Hamilton Medical AG: Bonaduz, Switzerland. 2021. Available online: https://www.hamiltonmedical.com/ja/Solutions/Ventilation-Cockpit-user-interface.html (accessed on 21 August 2021).

29. Hildebrand, P.W.; Rose, A.S.; Tiemann, J.K.S. Bringing Molecular Dynamics Simulation Data into View. Trends Biochem. Sci. 2019, 44, 902-913. [CrossRef] [PubMed]

30. Poncette, A.S.; Spies, C.; Mosch, L.; Schieler, M.; Weber-Carstens, S.; Krampe, H.; Balzer, F. Clinical Requirements of Future Patient Monitoring in the Intensive Care Unit: Qualitative Study. JMIR Med. Inform. 2019, 7, e13064. [CrossRef] [PubMed]

31. Blackler, A.; Popovic, V.; Mahar, D. Investigating users' intuitive interaction with complex artefacts. Appl. Ergon. $2010,41,72-92$. [CrossRef] [PubMed]

32. Pfarr, J.; Ganter, M.T.; Spahn, D.R.; Noethiger, C.B.; Tscholl, D.W. Avatar-Based Patient Monitoring With Peripheral Vision: A Multicenter Comparative Eye-Tracking Study. J. Med. Internet Res. 2019, 21, e13041. [CrossRef] [PubMed] 